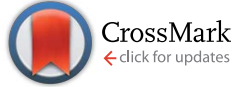

Cite this: Soft Matter, 2015, 11, 1562

\title{
Long-ranged and soft interactions between charged colloidal particles induced by multivalent coions
}

\author{
F. Javier Montes Ruiz-Cabello, ${ }^{a}$ Mohsen Moazzami-Gudarzi, ${ }^{a}$ Magdalena Elzbieciak- \\ Wodka, $\dagger^{\mathrm{a}}$ Plinio Maroni, ${ }^{\mathrm{a}}$ Christophe Labbez, ${ }^{\mathrm{b}}$ Michal Borkovec ${ }^{\mathrm{a}}$ and Gregor Trefalt ${ }^{\star a}$
}

Forces between charged particles in aqueous solutions containing multivalent coions and monovalent counterions are studied by the colloidal probe technique. Here, the multivalent ions have the same charge as the particles, which must be contrasted to the frequently studied case where multivalent ions have the opposite sign as the substrate. In the present case, the forces remain repulsive and are dominated by the interactions of the double layers. The valence of the multivalent coion is found to have a profound influence on the shape of the force curve. While for monovalent coions the force profile is exponential down to separations of a few nanometers, the interaction is much softer and longer-ranged in the presence of multivalent coions. The force profiles in the presence of multivalent coions and in the mixtures of monovalent and multivalent coions can be accurately described by Poisson-Boltzmann theory. These results are accurate for different surfaces and even in the case of highly charged particles. This behavior can be explained by the fact that the force profile follows the near-field limit to much larger distances for multivalent coions than for monovalent ones. This limit corresponds to the conditions with no salt, where the coions are expelled between the two surfaces.

Received 12th November 2014
Accepted 2nd January 2015

DOI: $10.1039 / \mathrm{c} 4 \mathrm{sm} 02510 \mathrm{e}$

www.rsc.org/softmatter potential (CP) might be inaccurate, and a more detailed description of charge regulation effects may be necessary. ${ }^{\mathbf{1 3 , 2 3 - 2 5}}$ The easiest way to incorporate such regulation effects is the constant regulation (CR) approximation. This approximation assumes a constant capacitance of the inner layer, and introduces only one additional parameter. ${ }^{23,26,27}$ The PB theory fails at smaller distances or higher salt levels, where one must consider additional interactions, especially van der Waals or hydration forces. ${ }^{11,14,15}$

Forces between charged surfaces in solutions containing multivalent ions came into focus more recently. ${ }^{1,8,28-36}$ The existing studies support the picture that the PB theory provides a reasonably accurate description of the forces, at least at distances larger than several nanometers and in sufficiently dilute solutions. They further confirm that multivalent counterions interact strongly with charged surfaces. ${ }^{29,32,33,37}$ In this commonly studied case, multivalent ions are oppositely charged than the surface, and therefore they adsorb strongly. Thereby, they reduce the surface charge density, eventually to the point that the surface undergoes a charge reversal. Multivalent ions may also induce additional attractive forces, which can make the total force stronger than the van der Waals force..$^{\mathbf{3 0 - 3 3}}$ These additional attractions are likely related to ion correlation effects. ${ }^{35,38-40}$ These effects are not treated in the classical mean-field PB theory, but they could also be responsible for adsorption of multivalent ions. ${ }^{29,41,42}$ 
On the other hand, multivalent coions adsorb onto surfaces only weakly or not at all. ${ }^{37}$ In this case, the charge of the multivalent ions has the same sign as the charge of the surface. The strong electrostatic repulsion leads to a depletion of these ions from the surface, and to a modification of the structure of the diffuse layer. ${ }^{\mathbf{4 3 4}}$ This situation has not been much investigated experimentally so far. ${ }^{7,8,37}$

The question whether the decay length of the double layer forces might deviate from the Debye length was equally raised. ${ }^{7,8,45,46}$ While PB theory predicts that double layer forces decay with the Debye length at large distances, theoretical studies of charge renormalization effects suggest that this decay length might be different, especially in asymmetric electrolytes. ${ }^{45,46}$ The currently available experimental results indicate that the measured screening lengths agree with the Debye length within experimental error, especially when complexation in solution is being considered..$^{5,7,8,32,33}$ One should realize, however, that the theoretically predicted deviations from the Debye length are relatively small, and probably within experimental error.

Force measurements involving multivalent ions almost exclusively involved multivalent counterions, meaning that the ions are oppositely charged as the surface. Multivalent coions that are equally charged as the surface were hardly addressed. We are aware only of a limited number of studies, where these conditions were realized, ${ }^{7,8,37}$ but these studies did not analyze the respective force profiles in any detail. This situation prompted us to reinvestigate these conditions more carefully. We show that multivalent coions may induce unusually soft and long-ranged double layer forces, which can be accurately described by PB theory.

\section{Poisson-Boltzmann (PB) theory}

Electric double layer forces between colloidal particles can be analyzed by the classical PB theory. ${ }^{21,23,47,48}$ This approach treats all ions as point charges in a dielectric continuum. Their interactions are described in a mean-field fashion, whereby ion-ion correlations are being neglected. When the particles are sufficiently large, the situation can be simplified by calculating the net pressure $\Pi(h)$ between two identical planar surfaces versus the surface separation $h$. Free energy per unit area can be then obtained by integrating the pressure profile, and the force between two particles follows from the Derjaguin approximation, which involves the effective radius. ${ }^{21}$ In the case of two spherical particles of the same size, the effective radius is half of the particle radius.

The net pressure can be calculated by solving the PB equation, which defines the electric potential $\psi(x)$ as a function of the position $x$, whose origin is taken at the mid-plane of the two surfaces located at $x= \pm h / 2$. When the solution contains different ions of number concentrations $c_{i}$ and valence $z_{i}$, the PB equation reads ${ }^{23,47}$

$$
\frac{\mathrm{d}^{2} \psi}{\mathrm{d} x^{2}}=-\frac{q}{\varepsilon_{0} \varepsilon} \sum_{i} z_{i} c_{i} \mathrm{e}^{-z_{i} \beta q \psi},
$$

where $q$ is the elementary charge, $\varepsilon_{0}$ the dielectric permittivity of vacuum, $\varepsilon$ the dielectric constant, and $\beta=1 /(k T)$ the inverse thermal energy. The latter relationship defines $T$ as the absolute temperature and $k$ as the Boltzmann constant. We use $\varepsilon=80$ as appropriate for water at room temperature. The solution of the PB equation is found within the constant regulation (CR) approximation..$^{23,45}$ In the $\mathrm{CR}$ approximation, each surface is characterized by the diffuse layer potential $\psi_{\mathrm{D}}$ and the regulation parameter $p$. The diffuse layer potential can be equally expressed in terms of the diffuse layer charge density $\sigma$. The regulation parameter represents the generalization of the constant charge (CC, $p=1)$ and constant potential (CP, $p=0$ ) boundary conditions. ${ }^{23,47}$ In general, the regulation parameter can also become negative. ${ }^{49}$

We assume that the solution contains a mixture of strong monovalent $(1: 1)$ and multivalent $(1: z$ or $z: 1)$ salts of known concentrations from which the respective ionic concentrations $c_{i}$ can be evaluated. The net pressure is the difference between the pressure in the slit and the bulk pressure, and in the symmetric situation the former is obtained from the electric potential at the midplane $\psi_{\mathrm{M}}=\psi(0){ }^{21,47}$ The midplane potential is calculated from the $\mathrm{PB}$ equation numerically subject to the boundary condition $\mathrm{d} \psi / \mathrm{d} x=0$ at $x=0$. To gain better insight into the different regimes, we further investigate two asymptotic solutions, namely the far-field and the near-field. The far-field regime reflects large separations and is equivalent to DH theory, where the solution composition enters only through the Debye length. The near-field regime describes small separations, where only the counterions neutralizing the surface charge contribute. This regime is equivalent to conditions without added salt.

\section{Far-field regime}

At large separations, the electric potentials are small, and thus the $\mathrm{PB}$ equation can be linearized, leading to the $\mathrm{DH}$ equation

$$
\frac{\mathrm{d}^{2} \psi}{\mathrm{d} x^{2}}=\kappa^{2} \psi
$$

where $\kappa$ is the inverse Debye length defined as

$$
\kappa^{2}=\frac{2 q^{2} I}{\varepsilon_{0} \varepsilon k T}
$$

and $I$ is the ionic strength

$$
I=\frac{1}{2} \sum_{i} z_{i}^{2} c_{i}
$$

which is also expressed as a number concentration. The $\mathrm{DH}$ equation can be solved analytically leading to the result

$$
\Pi=2 \varepsilon_{0} \varepsilon \kappa^{2} \psi_{\mathrm{eff}}^{2} \mathrm{e}^{-\kappa h}
$$

This relationship reflects the PB situation at larger separations, but $\psi_{\text {eff }}$ depends on the surface potential in a non-linear fashion with the limiting behavior

$$
\psi_{\text {eff }}=\left\{\begin{array}{cl}
\psi_{\mathrm{D}} & \text { for } \psi_{\mathrm{D}} \rightarrow 0 \\
\alpha k T / q & \text { for } \psi_{\mathrm{D}} \rightarrow \infty
\end{array}\right.
$$


The above relationship applies when the potential is positive, otherwise the appropriate negative signs must be introduced. For pure electrolytes, the reduced values for the saturation potential $\alpha$ are known, ${ }^{\mathbf{4 3 4 4}}$ and they are summarized for multivalent coions in Table 1 . The characteristic feature is that these values strongly increase with valence. Note that the far-field regime is independent of the boundary conditions.

\section{Near-field regime}

When the distance between the surfaces is small, the coions are expelled, and their charge is fully neutralized by the remaining counterions. This situation corresponds to no added salt, and the $\mathrm{PB}$ equation simplifies to ${ }^{\mathbf{4 2 - 4 4}}$

$$
\frac{\mathrm{d}^{2} \psi}{\mathrm{d} x^{2}}=\frac{q c}{\varepsilon_{0} \varepsilon} \mathrm{e}^{\beta q \psi},
$$

where we assume that the surface is positively charged, and that the counterions are monovalent anions of concentration $c$. The case with the opposite signs of the charge is obtained by replacing $q$ by $-q$. The solution of this equation reads ${ }^{2,50}$

$$
\psi=\psi_{\mathrm{M}}-\frac{1}{q \beta} \ln \cos ^{2}\left(\frac{x \gamma}{\lambda}\right),
$$

where $\gamma=\mathrm{e}^{\beta q\left(\psi_{\mathrm{M}}-\psi_{\mathrm{D}}\right) / 2}$ and

$$
\lambda=\frac{2 \varepsilon_{0} \varepsilon}{\beta q \sigma},
$$

is the Gouy-Chapman length. The net pressure is now given by

$$
\Pi=k T c \mathrm{e}^{\beta q \psi_{\mathrm{M}}}
$$

and it is equal to the pressure in the slit, since the bulk pressure vanishes without salt. The pressure can be parameterized through the dimensionless quantity $\gamma$ defined above, namely

$$
\Pi=\frac{2 \varepsilon_{0} \varepsilon}{\beta^{2} q^{2} \lambda^{2}} \gamma^{2}
$$

By invoking the potential profile given by eqn (8) and using the constant regulation boundary conditions one finds that the pressure is determined by

$$
p[\gamma \tan (\ell \gamma)-1]+(1-p) \ln \left[\frac{\gamma}{\cos (\ell \gamma)}\right]=0
$$

where $\ell=h /(2 \lambda)$ and $p$ is the regulation parameter. When one analyses eqn (12) for large separation distances, the pressure profile again becomes independent of the boundary conditions, and reads

Table 1 Far-field limit for the saturation values of effective potentials for $z$ : 1 electrolytes containing multivalent coions

\begin{tabular}{lccccc}
\hline$z$ & 1 & 2 & 3 & 4 & 5 \\
\hline$\alpha$ & 4 & 6 & 8.707 & 12.314 & 17.337 \\
$\psi_{\text {eff }}(\mathrm{mV})$ & 102.7 & 154.0 & 223.5 & 316.1 & 445.0
\end{tabular}

$$
\Pi=\frac{2 \pi^{2} \varepsilon \varepsilon_{0}}{\beta^{2} q^{2}} \frac{1}{h^{2}}
$$

The free energy can be calculated through integration of the pressure, but the appropriate integration constant must be inferred from the solution of the full PB equation.

\section{Experimental}

\section{Materials}

Two different types of polystyrene latex particles were used, namely sulfate-terminated latex (SL) and amidine-terminated latex $(\mathrm{AL})$, and they were obtained from Invitrogen. Further experiments were carried out with silica particles obtained from Bangs Laboratories Inc., USA. Table 2 summarizes the mean diameters and polydispersities as determined by the manufacturer.

Experiments were carried out at $22 \pm 2{ }^{\circ} \mathrm{C}$ in solutions of $\mathrm{KCl}$, $\mathrm{K}_{2} \mathrm{SO}_{4}, \mathrm{LaCl}_{3}, \mathrm{~K}_{3} \mathrm{Fe}(\mathrm{CN})_{6}$, and $\mathrm{K}_{4} \mathrm{Fe}(\mathrm{CN})_{6}$ in Milli-Q water. For the SL particles pH 5.6 was used, while for $\mathrm{AL}$ particles the solutions were adjusted to $\mathrm{pH} 4.0$ with $\mathrm{HCl}$, and for the silica particles with $\mathrm{KOH}$ to $\mathrm{pH}$ 10.0. The specific $\mathrm{pH}$ values were chosen in order to increase the magnitude of the surface charge density, while keeping the ionic strength low. Some experiments were also performed in solutions of aliphatic polyamine, namely of 3,6,9,12-tetraazatetradecane-1,14-diamine (N6) with chemical formula $\mathrm{H}_{2} \mathrm{~N}-\left(\mathrm{CH}_{2}-\mathrm{CH}_{2}-\mathrm{NH}\right)_{4}-\mathrm{CH}_{2}-\mathrm{CH}_{2}-\mathrm{NH}_{2}$, obtained from Sigma-Aldrich (St. Louis, USA). At pH 4.0, $\mathrm{La}^{3+}$ does not hydrolyze, and N6 has the +4 cation as the predominant species. $^{51}$ The experiments were carried out at ionic strengths between 1 and $3.1 \mathrm{mM}$. The ionic strength was chosen as low as possible, such that the low salt regime is well developed, while high enough, such that ions originating from the self-dissociation of water or carbonate dissolution remain negligible.

\section{Direct force measurements}

Forces between particles were measured with a closed-loop AFM (MFP-3D, Asylum Research) mounted on an inverted optical microscope (Olympus IX70). ${ }^{\mathbf{1 3 , 5 2}}$ The particles were attached on tip-less cantilevers (MikroMasch, HQ CSC37, without Al coating) and to the substrate. A pair of particles was centered laterally through optical fringes viewed in the optical microscope with an accuracy of about $100 \mathrm{~nm}$. For one pair of particles, we typically recorded about 100 vertical approachretraction cycles with an approach-retraction velocity of $300 \mathrm{~nm}$ $\mathrm{s}^{-1}$, a sampling rate of $5 \mathrm{kHz}$, and a cycle frequency of $0.5 \mathrm{~Hz}$. The contact point was obtained from the constant compliance region. The cantilever deflection recorded in the approach part was transformed into force profiles by means of the spring constant of the cantilever. The latter constant was measured by the method developed by Sader et al. ${ }^{53}$ which relies on the frequency response of the cantilever and its geometrical dimensions. Typical values of the spring constants were $0.1-$ $0.3 \mathrm{~N} \mathrm{~m}^{-1}$. These values agreed within about $10 \%$ with those determined through the thermal fluctuations of the cantilever. ${ }^{54}$ The forces were subsequently down-sampled to $150 \mathrm{~Hz}$ and 
Table 2 Particle and selected solution characteristics

\begin{tabular}{lllllrrr}
\hline Particles & Abbreviation & $\begin{array}{l}\text { Diameter } \\
(\mu \mathrm{m})\end{array}$ & $\begin{array}{l}\text { Polydispersity } \\
(\%)\end{array}$ & $\begin{array}{l}\text { RMS roughness } \\
(\mathrm{nm})\end{array}$ & $\begin{array}{l}\text { pH } \\
\left(\mathrm{mC} \mathrm{m}^{-2}\right)\end{array}$ \\
\hline Sulfate latex & $\mathrm{SL}$ & 3.0 & 4.1 & 0.8 & 5.6 & -9.3 \\
Amidine latex & $\mathrm{AL}$ & 0.95 & 3.6 & 0.5 & 4.0 & +7.5 \\
Silica & $\mathrm{SiO}_{2}$ & 5.2 & 10 & 1.4 & 10.0 & -4.4 & 0.64 \\
parameter $p$
\end{tabular}

averaged over the different approach curves, leading to a force resolution of about $1 \mathrm{pN}$. All measurements were repeated with at least three pairs of different particles, and they were well reproducible. The relative error between the resulting double layer potentials, which was determined by fitting the force curves for every pair of particles under the same conditions, was below $15 \%$.

Latex particles were mounted in solution within the AFM fluid cell. A glass plate fitting the fluid cell was used as the substrate. The glass plate and cantilevers were cleaned overnight in a piranha solution, which consists of $\mathrm{H}_{2} \mathrm{SO}_{4} 98 \%$ and $\mathrm{H}_{2} \mathrm{O}_{2} 30 \%$ mixed in ratio $3: 1$. They were further rinsed with water, dried, and cleaned in an air-plasma for $20 \mathrm{~min}$. Silanization of the plate and cantilevers was carried out overnight in an evacuated container aside two drops of $20 \mu \mathrm{L}$ of 3-ethoxydimethylsilylpropylamine and $100 \mu \mathrm{L}$ of (3-glycidoxypropyl) dimethylethoxysilane. The silanized plate was then introduced into the AFM fluid cell, and the cantilever into the cantilever holder. Before the experiments, the stock latex particle suspension was purified by dialysis against Milli-Q (Millipore) until the conductivity of the dialysate reached the value of the pure water. The particle suspensions were prepared in the respective electrolyte solutions at a particle concentration of $80 \mathrm{mg} \mathrm{L}^{-1}$, and injected into the fluid cell. The particles were allowed to settle for a few hours, and the cell was then rinsed with the pure electrolyte solution. By pressing the particle against the substrate with the cantilever, one could firmly attach a particle to the cantilever. For the latex particles, the constant compliance region could be identified after the jump-in in the load range of 5-10 $\mathrm{nN}$ with an absolute accuracy of about $0.3 \mathrm{~nm}$.

The silica particles were attached by sintering in the dry state. Cantilevers were cleaned in air-plasma for 5 minutes. Few silica particles were placed on a glass slide and tiny drops of about $5 \mu \mathrm{L}$ of glue (Araldite 2000+) were deposited in their proximity. The cantilever was mounted in the AFM, brought in contact with the glue with the translation stage, and used to pick up the particle. The cantilever was removed from the AFM, and placed into an oven at $1150{ }^{\circ} \mathrm{C}$ for $3 \mathrm{~h}$. The same particles were attached to the glass slide sealing the AFM fluid cell as follows. The slide was cleaned with piranha solution for 2 hours, then rinsed with water, and dried under a stream of nitrogen. Subsequently, particles were spread onto a square quartz slide of $19 \mathrm{~mm}$ (Edmund Optics), and then sintered at $1150{ }^{\circ} \mathrm{C}$ for 3 hours. The reverse side of the slide with the sintered particles was glued (Pattex 100\% Repair Gel) onto a glass slide sealing the AFM cell. After sintering, the substrate and the cantilevers were again cleaned in an air-plasma. This procedure leads to a firm attachment of the particles to the substrate and to the cantilever, while completely removing traces of the glue and of any organic impurities. For the silica particles, the contact point was identified at a load of about $5 \mathrm{nN}$. The precision of the contact position is inferior to the latex particles, but typically below $1 \mathrm{~nm}$.

\section{Particle roughness}

The root mean square (RMS) roughness of the particles was measured by AFM imaging. The latex particles were deposited for about 1 hour on a piranha-cleaned and silanized glass slide with dimensions about $1 \mathrm{~cm} \times 1 \mathrm{~cm}$. These slides were silanized overnight in an evacuated glass container aside a $50 \mu \mathrm{L}$ drop of 3-ethoxydimethylsilylpropylamine (for SL) or (3-glycidoxypropyl)dimethylethoxysilane (for AL). After the deposition of the particles, the substrate was rinsed in $10 \mathrm{mM} \mathrm{KCl}$ solution. The images were recorded in liquid with a Cypher AFM instrument (Asylum Research, Santa Barbara, USA) with BioLever Mini cantilevers (BL-AC40TS, Olympus, Japan). They had a nominal tip radius of $<9 \mathrm{~nm}$ and a resonance frequency of around $30 \mathrm{kHz}$ in water. The scan rate was $2.0 \mathrm{~Hz}$, the scan size $0.5 \mu \mathrm{m} \times 0.5 \mu \mathrm{m}$, and the free oscillation amplitude (FOA) $20 \mathrm{~nm}$. The set-point was fixed at around $70 \%$ of the FOA.

The roughness of the silica particles was determined as follows. The particles sintered onto a quartz slide, which was previously cleaned with piranha solution, rinsed with water, and finally treated in air plasma for $20 \mathrm{~min}$. The particles were imaged in air with the MFP-3D in amplitude modulation mode. Silicon cantilevers (OMCL-AC240TS, Olympus) with a nominal tip radius $<10 \mathrm{~nm}$ and a resonance frequency of about $70 \mathrm{kHz}$ were used. Images were acquired with a scan rate of $2 \mu \mathrm{m} \mathrm{s}^{-1}$, an FOA of about $40 \mathrm{~nm}$, and a set point around $80 \%$ of the FOA. The roughness was determined for an area of $1 \mu \mathrm{m} \times 1 \mu \mathrm{m}$. The RMS roughness of the particles is summarized in Table 2 . These values are $<1 \mathrm{~nm}$ for the latex particles, and $1.4 \mathrm{~nm}$ for the silica particles. All particles used can be thus considered to be smooth for the relevant surface separations considered.

\section{Results and discussion}

We present direct force measurements between pairs of similar colloidal particles in aqueous solutions containing multivalent coions. These multivalent ions have the same sign of the charge as the surface, and they do not adsorb on the surface. With respect to the monovalent situation, however, one obtains much softer and long-ranged force profiles. Several types of 
particles and different coions are compared with PB theory, which captures the observed profiles very well.

\section{Pressure profiles}

Let us first discuss pressure profiles between two charged plates calculated with PB theory. These profiles are shown in Fig. 1, and they reveal the relevant features more clearly than the force profiles discussed below. At larger distances, the profiles decay exponentially as one expects from the far-field linearized DH solution. This decay is evident from the linear dependence in the semi-logarithmic representation. As the separation decreases, the coions are progressively being expelled between the plates. When their concentration becomes negligible, the pressure profile follows the near-field solution of the PB equation. This solution assumes that the charge of the plates is fully neutralized by counterions, which corresponds to the situation of no added salt.

Fig. $1 \mathrm{~b}$ shows the calculated force profiles for plates of a surface change density of $-10 \mathrm{mC} \mathrm{m}^{-2}$ in a solution of an ionic
Monovalent Coions
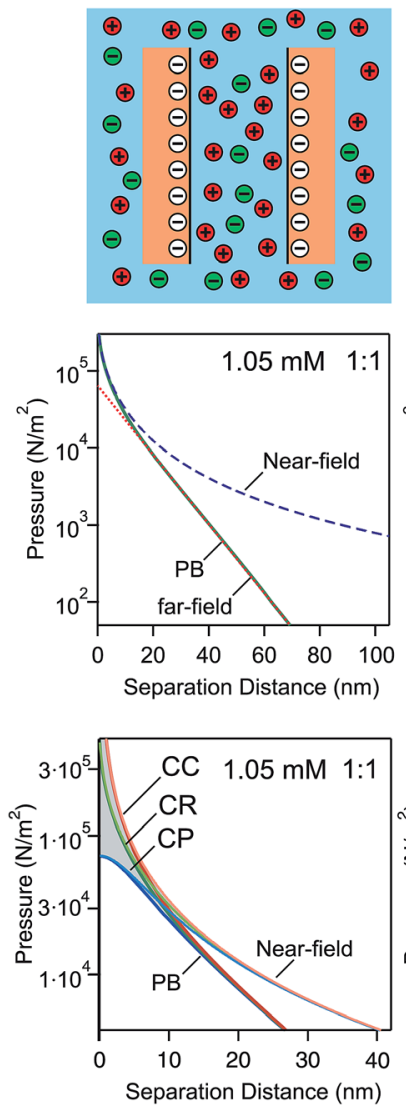

Multivalent Coions
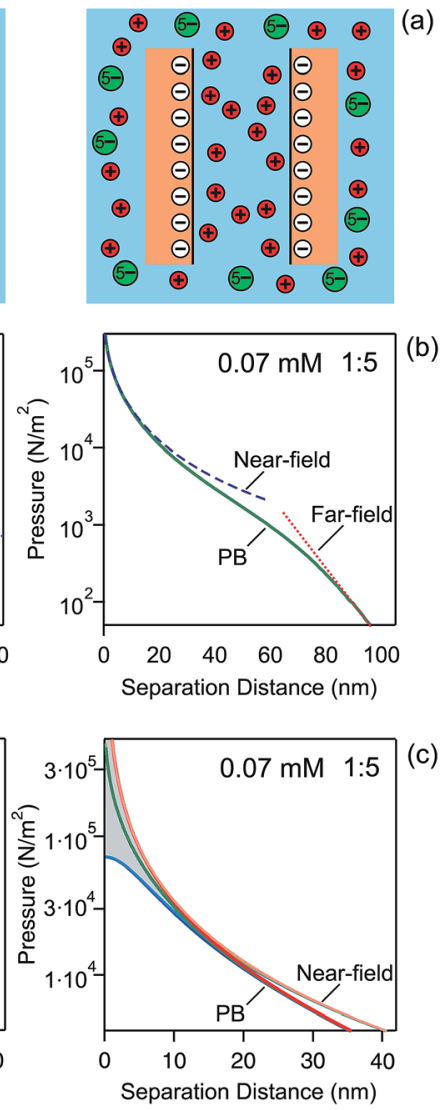

Fig. 1 (a) Schematic presentation of expulsion of the multivalent coions between the charged walls. (b) Net pressure between charged plates in the presence of $1.05 \mathrm{mM} 1: 1$ and $0.05 \mathrm{mM} 1: 5$ electrolyte calculated with the PB theory. The near and far-field asymptotes are denoted by dashed lines. (c) The effect of the CC, CR, and CP boundary conditions for the same surfaces. Near-field solution is also shown. A surface charge density of $-10 \mathrm{mC} \mathrm{m} \mathrm{m}^{-2}$ and regulation parameter $p=0.5$ are used throughout. lonic strength is $1.05 \mathrm{mM}$. strength of $1.05 \mathrm{mM}$. This surface charge density corresponds to the diffuse layer potentials $-87 \mathrm{mV}$ and $-114 \mathrm{mV}$ in $1: 1$ and $1: 5$ electrolytes, respectively. The left panel shows the familiar situation of monovalent $1: 1$ salt. The pressure profile is dominated by the exponential far-field DH profile down to separations of a few nanometers. The effective potential is $-69 \mathrm{mV}$, which is about half of the saturation value. The nearfield profile sets in at small separation distances only. For a pentavalent 1:5 electrolyte, the profile is much softer and longer-ranged, and features a sigmoidal shape. At larger distances the pressure is again exponential, but this dependence only sets in at relatively large distances, around $80 \mathrm{~nm}$ here. The effective potential is now $-293 \mathrm{mV}$, which is still substantially below the saturation value. At smaller distances, the pressure follows the near-field regime, but this regime now sets in at much larger distances than for the monovalent ones, about $30 \mathrm{~nm}$ in this case. This wider region of validity is related to the larger magnitude of the effective potential. This larger value results from the fact that the multivalent coions get expelled from the slit at larger distances than the monovalent ones. This difference is a consequence of the higher charge of the multivalent ions, which leads to their higher electrostatic energy when they are situated between the plates.

The details of the pressure profiles at smaller distances are shown in Fig. 1c. At small separations, the effect of boundary conditions is substantial. The CC conditions, characterized by the regulation parameter $p=1$, result in the strongest repulsion by the double layer forces. Regulation effects are reflected by smaller regulation parameters and they lead to the decrease of the strength of the repulsion, as illustrated with $p=0.5$ and $p=0$ (CP conditions). At larger distances, the effect of boundary conditions disappears, and the near-field solution converges to eqn (13). The near-field solution reproduces the full PB calculations at small distances. However, its region of validity is much wider for the multivalent coions than for the monovalent ones.

Characteristic length scales in the far-field and near-field regimes are the Debye length $\kappa^{-1}$ and Gouy-Chapman length $\lambda$. In this example, we approximately have $\kappa^{-1}=9.5 \mathrm{~nm}$ and $\lambda=$ $3.6 \mathrm{~nm}$. The Debye length reflects the range of the exponential decay at larger distances, while the Gouy-Chapman length measures the thickness of the layer containing counterions only.

\section{Sulfate latex particles}

Let us first discuss the effect of multivalent coions on experimentally measured force profiles between negatively charged SL particles of $3 \mu \mathrm{m}$ in diameter. Fig. 2 compares forces in $1.0 \mathrm{mM}$ $\mathrm{KCl}$ solutions and $0.1 \mathrm{mM} \mathrm{K}_{4} \mathrm{Fe}(\mathrm{CN})_{6}$ solutions. These strong electrolytes fully dissociate into $\mathrm{Fe}(\mathrm{CN})_{6}{ }^{4-}$ or $\mathrm{Cl}^{-}$, which are the coions, $\mathrm{K}^{+}$being the counterion. The ionic strength of both solutions is the same, namely $1.0 \mathrm{mM}$. The force profile in the monovalent electrolyte shown in Fig. 2a is exponential down to a few nanometers, as was the case for the pressure. The profile in the presence of multivalent coions shown in Fig. $2 \mathrm{~b}$ is much softer, and features a sigmoidal shape. In this case, the 


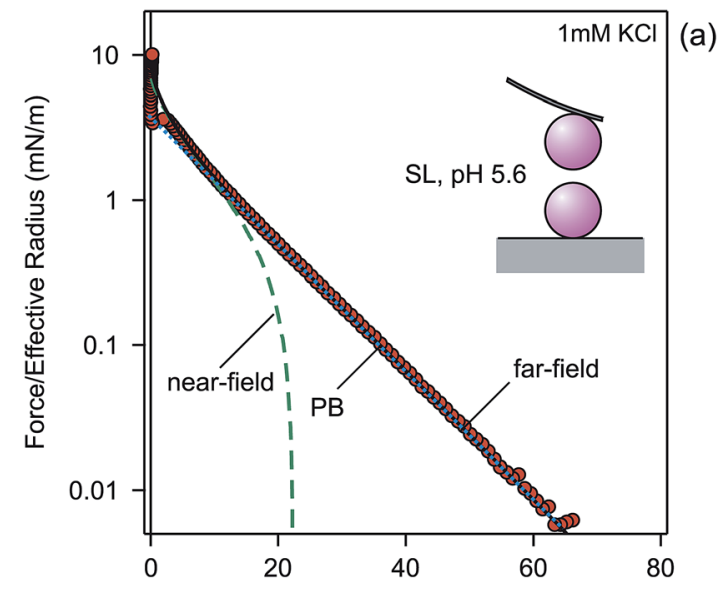

Separation Distance $(\mathrm{nm})$

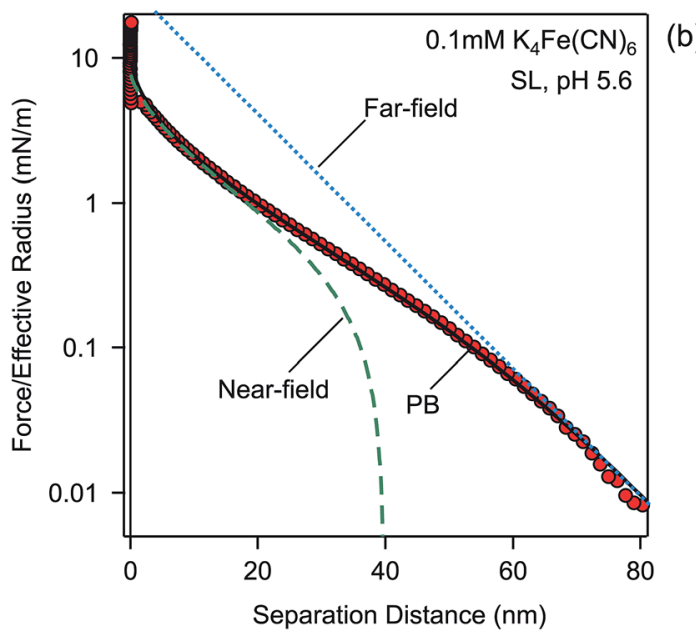

Fig. 2 Forces between SL particles in (a) $1 \mathrm{mM} \mathrm{KCl}$ and (b) $0.1 \mathrm{mM}$ $\mathrm{K}_{4} \mathrm{Fe}(\mathrm{CN})_{6}$ at $\mathrm{pH}$ 5.6. Points designate the experiments, while solid lines are PB calculations with a surface charge density of $-9.3 \mathrm{mC} \mathrm{m}^{-2}$ and regulation parameter $p=0.64$. The far-field and near-field asymptotes are presented with dashed lines.

exponential dependence sets in only around $60 \mathrm{~nm}$. In both cases, one observes a jump-in close to contact, which occurs due to additional van der Waals attraction and hydrophobic forces.

The measured force profiles can be described with PB theory perfectly well. By fitting the force profile measured in the $\mathrm{KCl}$ solution, we extract the surface charge density $\sigma=-9.3 \pm 0.1$ $\mathrm{mC} \mathrm{m}^{-2}$ and a regulation parameter $p=0.64 \pm 0.02$ (see also Table 2). The calculation uses the fixed analytical $\mathrm{KCl}$ concentration of $1.0 \mathrm{mM}$. When this concentration would be adjusted, the fitted concentration will remain within $5 \%$ of this value. We now keep the same surface parameters, and predict the force profile in $0.10 \mathrm{mM} \mathrm{K} \mathrm{K}_{4} \mathrm{Fe}(\mathrm{CN})_{6}$ solution with the PB theory. Again, the analytical concentration is being fixed. The calculation reproduces the sigmoidal shape of the measured force curve extremely well. The fact that the force profiles are consistent with the same surface charge density for monovalent and multivalent coions suggests that the multivalent ions adsorb onto the surface very weakly, or not at all. The lack of adsorption is obviously related to the strong electrostatic repulsion between multivalent coions and the surface.
The reason for the different appearance of the force curves is related to the fact that multivalent coions are being expelled from the gap between the surfaces at much larger separations than the monovalent ones. Therefore, the near-field solution provides a good description of the force profile to larger distances, albeit not as large as was the case for the pressure profile. The sigmoidal dependence is reinforced by the larger magnitude of the effective potential entering the far-field profile for the multivalent salts. For $\mathrm{KCl}$, the effective potential is $\psi_{\text {eff }}=-67 \mathrm{mV}$, while for $\mathrm{K}_{4} \mathrm{Fe}(\mathrm{CN})_{6}$ one has $\psi_{\text {eff }}=-176 \mathrm{mV}$. The magnitude of these values is still substantially below the saturation values given in Table 1. At the same time, the force profile must converge into the near-field profile at small distances. While for $\mathrm{KCl}$, the far-field asymptote lies below the near-field profile, for $\mathrm{K}_{4} \mathrm{Fe}(\mathrm{CN})_{6}$ the far-field asymptote lies above it, and thus the force curve makes the sigmoidal transition converge into the near-field profile.

Further force measurements with other solution compositions confirm that PB theory describes the force profiles quantitatively. Fig. 3 shows measurements in $\mathrm{K}_{4} \mathrm{Fe}(\mathrm{CN})_{6}$ solutions of varying concentrations. Thereby, the charge density and regulation properties of the surface were kept at the previous values (Table 2) and the solution concentrations were fixed to the known analytical concentrations of the solutions. These calculations contain no adjustable parameters, and provide very satisfactory results.

Fig. 4a shows another test of the PB theory, as these experiments were carried out in mixtures of $\mathrm{KCl}$ and $\mathrm{K}_{4} \mathrm{Fe}(\mathrm{CN})_{6}$ solutions at a constant ionic strength of $1.0 \mathrm{mM}$. The calculations rely on the solution of the PB equation for mixed electrolytes, and they predict the observed force profiles very well. Again, no adjustable parameters were used.

Fig. $4 \mathrm{~b}$ summarizes the effect of the valence of different coions. These experiments were carried out in solutions of $\mathrm{KCl}$, $\mathrm{K}_{2} \mathrm{SO}_{4}, \mathrm{~K}_{3} \mathrm{Fe}(\mathrm{CN})_{6}$, and $\mathrm{K}_{4} \mathrm{Fe}(\mathrm{CN})_{6}$, thus covering valences of

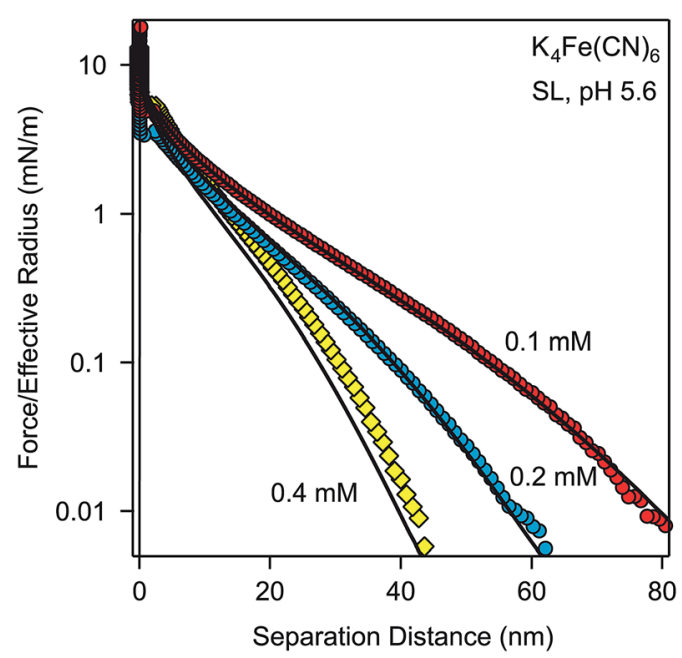

Fig. 3 Forces between SL particles in different concentrations of $\mathrm{K}_{4} \mathrm{Fe}(\mathrm{CN})_{6}$ at $\mathrm{pH}$ 5.6. Points designate the experiments, while solid lines are predictions with the PB theory with a surface charge density of $-9.3 \mathrm{mC} \mathrm{m}^{-2}$ and regulation parameter $p=0.64$. 

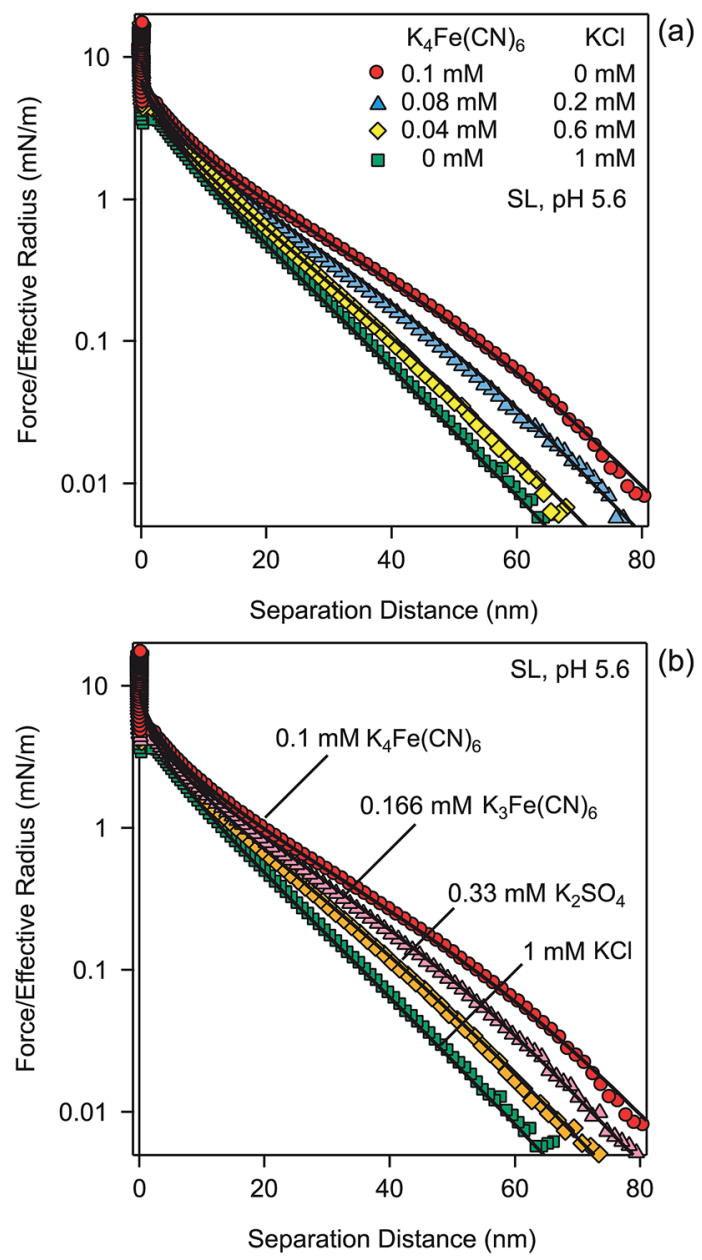

Fig. 4 (a) Forces between SL particles in a mixture of $\mathrm{KCl}$ and $\mathrm{K}_{4} \mathrm{Fe}(\mathrm{CN})_{6}$ at $\mathrm{pH} 5.6$ and fixed ionic strength of $1 \mathrm{mM}$. (b) Forces in $\mathrm{KCl}$, $\mathrm{K}_{2} \mathrm{SO}_{4}, \mathrm{~K}_{3} \mathrm{Fe}(\mathrm{CN})_{6}$, and $\mathrm{K}_{4} \mathrm{Fe}(\mathrm{CN})_{6}$ solutions at $\mathrm{pH} 5.6$ and constant ionic strength of $1 \mathrm{mM}$. Points designate the experiments, while solid lines are predictions of the PB theory with a surface charge density of $-9.3 \mathrm{mC} \mathrm{m}^{-2}$ and regulation parameter $p=0.64$.

coions of $-1,-2,-3$, and -4 . The ionic strength was fixed to 1.0 $\mathrm{mM}$. The corresponding predictions of the $\mathrm{PB}$ theory, which contain no adjustable parameters, are again in excellent agreement with experiment.

In all cases, a fixed value of surface charge density satisfactorily describes the forces, which implies that the ions involved do not adsorb onto the particles. This behavior is characteristic for multivalent coions, and is in contrast to multivalent counterions, which readily adsorb onto the surfaces and modify the surface charge or even induce charge reversal. ${ }^{29,32,33}$ However, the assumption of a fixed surface charge density leads to minor discrepancies, for example, for the highest concentration shown in Fig. 3. These discrepancies are probably related to small variations of the surface charge density and the surface regulation properties. These variations could be caused by weak adsorption of the ions involved onto the surface.

All calculations used the analytical concentrations. While the analytical concentrations are accurate within $<1 \%$, some modification might result from chemical decomposition of
$\mathrm{Fe}(\mathrm{CN})_{6}{ }^{4-}$ due to oxidation. We find that the analytical concentrations are consistent with the measured force profiles to an accuracy of about $<10 \%$. This agreement is comparable to previous studies that attempted to determine the salt concentrations from measured force profiles. ${ }^{5,7,8,32,33}$

\section{Amidine latex particles}

A similar set of experiments was carried out with positively charged AL particles of a diameter of $0.95 \mu \mathrm{m}$. For these particles, the cations are the coions, and for this reason we investigated solutions containing $\mathrm{La}^{3+}$ and $\mathrm{K}^{+}$. The forces in mixtures of $\mathrm{KCl}$ and $\mathrm{LaCl}_{3}$ solutions at a fixed ionic strength of $3.1 \mathrm{mM}$ are shown in Fig. 5a. One observes the same features as in the previously discussed case with the SL particles. In the monovalent salt, the decay is exponential of larger part of the accessible distance range, typically down to a few nanometers. For multivalent coions, the exponential decay sets in at larger

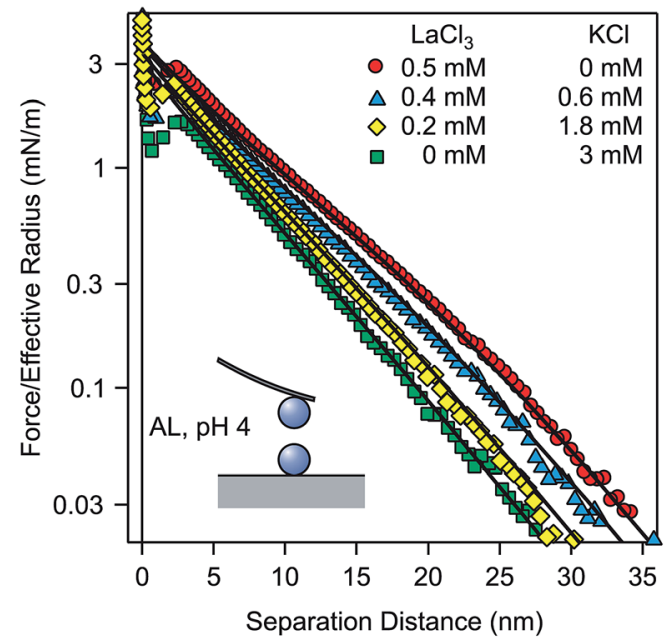

(a)

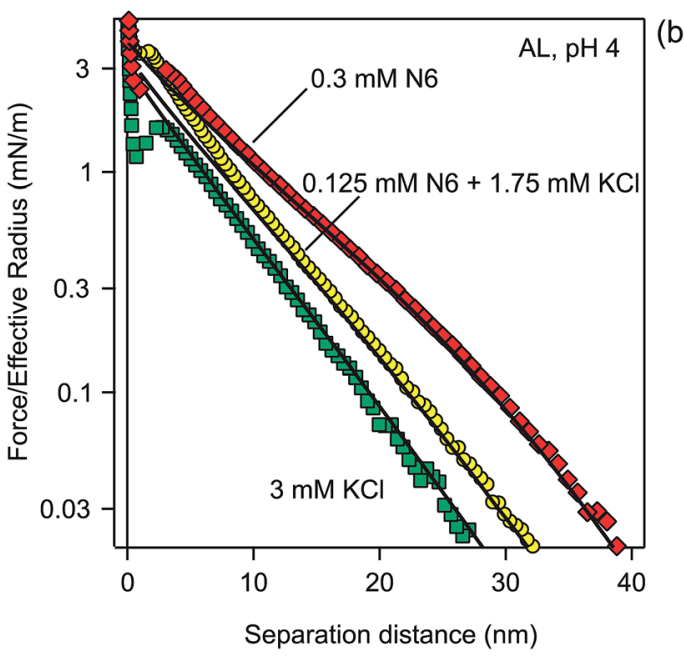

Fig. 5 (a) Forces between $\mathrm{AL}$ particles in a mixture of $\mathrm{KCl}$ and $\mathrm{LaCl}_{3}$ at $\mathrm{pH} 4$ and a fixed ionic strength of $3.1 \mathrm{mM}$. (b) Forces in the presence of $\mathrm{N} 6$ polyamine and its mixture with $\mathrm{KCl}$. Points designate the experiments, while solid lines are predictions with the PB theory with a surface charge density of $+7.5 \mathrm{mC} \mathrm{m}^{-2}$ and regulation parameter $p=$ 0.31. 
distances, at about $30 \mathrm{~nm}$. Again, the characteristic soft and sigmoidal force profile is observed. For the AL particles, one observes a more pronounced jump-in close to contact, which probably occurs since the hydrophobic forces are stronger for AL than for SL .

The surface properties of the particles were again determined by fitting the force profile in $\mathrm{KCl}$ solutions, whereby the solution concentrations were fixed to the analytical values. The respective surface charge density was $\sigma=+7.5 \pm$ $0.1 \mathrm{mC} \mathrm{m}^{-2}$ and the regulation parameter $p=0.31 \pm 0.02$ (Table 2). With these values, one can correctly predict the force profiles in all mixtures. These calculations contain again no adjustable parameters, since the analytical concentrations and the surface properties are kept fixed (Table 2). We have also fitted the electrolyte concentrations, and we found that they agreed within $10 \%$ with the analytical ones. A small discrepancy could also be related to the formation of $\mathrm{LaCl}^{2+}$ complexes in solution. ${ }^{33,55}$ The reason for the softer repulsion is again related to the more effective exclusion of the multivalent coions from the gap and to the larger effective potentials for the multivalent coions (Table 1). The near-field profile again remains a good approximation at much larger distances for multivalent coions than for monovalent ones.

Fig. 5b shows a similar effect with highly charged organic aliphatic polyamine. N6 is a linear amine, which does not ionize fully, and the prevalent species has a valence of +4 . The forces in the presence of $\mathrm{N} 6$ and its mixture with $\mathrm{KCl}$ are predicted with PB theory only by invoking the respective concentrations. Again the ionic strength is fixed to $3.1 \mathrm{mM}$. No parameter adjustment was made, as the same surface properties as before were used. Good agreement with the observed force profiles was found. The minor discrepancies could be related to weak adsorption onto the surface or due to the contribution of other valences,

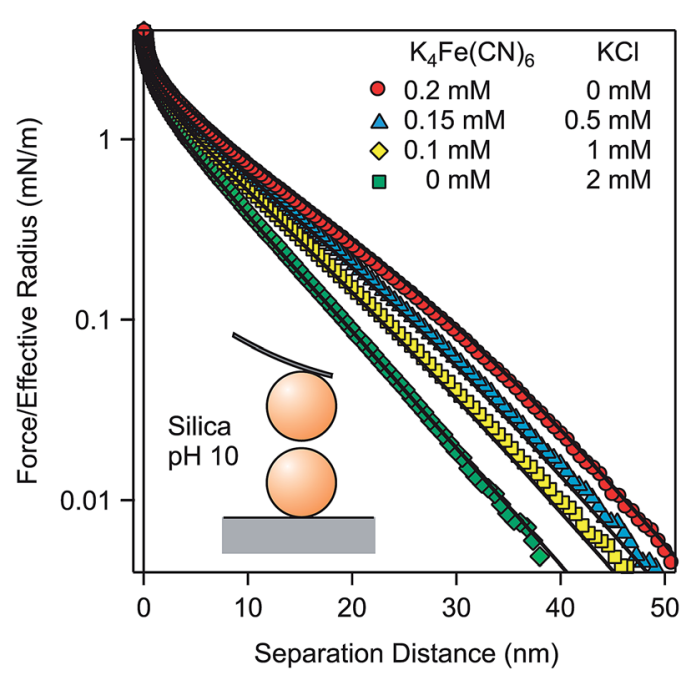

Fig. 6 Forces between silica particles in a mixture of $\mathrm{KCl}$ and $\mathrm{K}_{4} \mathrm{Fe}(\mathrm{CN})_{6}$ at $\mathrm{pH} 10$ and a fixed ionic strength of $2.1 \mathrm{mM}$. Points designate the experiments, while solid lines are predictions of $\mathrm{PB}$ theory with a surface charge density of $-4.4 \mathrm{mC} \mathrm{m}^{-2}$ and regulation parameter $p=0.66$. which occur in small concentrations due to the multi-step dissociation equilibria.

\section{Silica particles}

To confirm that the described effects are generic for various types of materials, forces between negatively charged silica particles of $5.2 \mu \mathrm{m}$ in diameter were measured in solutions of $\mathrm{K}_{4} \mathrm{Fe}(\mathrm{CN})_{6}, \mathrm{KCl}$, and their mixtures at an ionic strength of 2.1 $\mathrm{mM}$ (Fig. 6). As for the previously discussed systems, one observes softer profiles in the presence of multivalent coions. The main difference to the previous force profiles is that forces remain repulsive down to contact and that one cannot observe a jump-in at short distances. This feature is probably related to repulsive short-ranged hydration forces acting between silica surfaces..$^{17,19,20}$

Surface properties of silica were determined by fitting the force profile in $\mathrm{KCl}$ solution with $\mathrm{PB}$ theory. Thereby, the analytical salt concentration was used as before. From this fit, we obtain the surface charge density $\sigma=-4.4 \pm 0.1 \mathrm{mC} \mathrm{m}^{-2}$ and a regulation parameter $p=0.66 \pm 0.02$ (Table 2). These parameters are then used to predict the force profiles in $\mathrm{K}_{4} \mathrm{Fe}(\mathrm{CN})_{6}$ solutions and their mixtures with KCl. Since the appropriate salt concentrations were kept fixed, no adjustable parameters enter the calculation. The PB theory again describes the measured force profiles very well. The characteristic features observed for the force profiles in the presence of multivalent coions do not depend on the nature of the particles. The obtained surface charge density is well comparable to the published values measured for silica surfaces at $\mathrm{pH} \sim 10$ at low salt concentrations. ${ }^{\mathbf{1 7 - 2 0 , 5 6}}$ These values were determined by force measurements and they are in the range from -8 to $-4 \mathrm{mC} \mathrm{m}^{-2}$.

\section{Conclusions}

Direct force measurements were carried out with different types of negatively and positively charged colloidal particles in aqueous electrolyte solutions containing multivalent coions. In all situations studied, the multivalent ions have the same sign of charge as the particles. While these ions hardly adsorb onto such surfaces, they strongly modify the structure of the diffuse layer. As a consequence, one observes unusually softer and longer-ranged force profiles than for monovalent electrolytes. These profiles have a sigmoidal appearance in the popular semi-logarithmic representation and they can be described with PB theory for asymmetric electrolytes very well. These characteristic features reflect the fact that multivalent coions get expelled from the gap between the surfaces at larger distances than for monovalent ones. At short distances, the force profile converges to the near-field solution of the PB equation, which reflects the situation where the surface charge is neutralized by counterions only, as is the case without added salt. In the presence of multivalent coions, the near-field profile represents a good approximation up to larger separation distances than for monovalent coions. This fact is also reflected by the effective potentials entering the 
far-field $\mathrm{DH}$ theory, which are higher for the multivalent coions than for the monovalent ones. These findings demonstrate that the $\mathrm{PB}$ theory can accurately describe forces in the presence of multivalent coions even when surfaces are highly charged.

The measured force profiles reflect the analytical electrolyte concentrations very well. While the observed discrepancies are typically $<10 \%$, these deviations could also be related to complexation in solution or to a chemical decomposition of the ions. The present work confirms the findings of previous studies, ${ }^{7,8}$ which concluded that salt concentrations determined from direct force measurements are in agreement with the analytical ones. Systematic deviations between experimentally observed decay lengths in the far-field regime and the Debye lengths cannot be confirmed even in the presence of multivalent ions. While these deviations might exist, they are beyond the resolution of the surface force instruments currently in use.

The present work contributes to the current discussion on the role of multivalent ions in electrostatic interactions between charged surfaces. ${ }^{\mathbf{8} 29-34,36}$ Thereby, a major difference between multivalent counterions and multivalent coions must be stressed. Multivalent counterions strongly adsorb onto charged surfaces, and they may eventually lead to overcharging. Multivalent coions do not adsorb onto such surfaces, or eventually only weakly. Therefore, they do not affect the surface charge, but they strongly modify the structure of the electric double layer and the resulting interaction forces. Therefore, addition of multivalent coions provides a new means of tuning repulsive double layer forces. This possibility might be of relevance in colloidal self-assembly, where delicate balance between interactions must be achieved in order to obtain the regular particle arrangements. ${ }^{57,58}$ In this respect, a more detailed understanding how multivalent ions influence forces between dissimilar surfaces would be desirable as well.

\section{Acknowledgements}

This research was supported by the Swiss National Science Foundation, University of Geneva, COST Action CM1101, and the Swiss Secretariat of Education and Research. We acknowledge helpful discussions with Jordi Faraudo and Peter Kralchevsky.

\section{References}

1 R. M. Pashley and J. N. Israelachvili, J. Colloid Interface Sci., 1984, 97, 446-455.

2 J. Israelachvili, Intermolecular and Surface Forces, Academic Press, London, 2nd edn, 1992.

3 T. Baimpos, B. R. Shrestha, S. Raman and M. Valtiner, Langmuir, 2014, 30, 4322-4332.

4 H. J. Butt, B. Cappella and M. Kappl, Surf. Sci. Rep., 2005, 59, 1-152.

5 A. Tulpar, V. Subramanian and W. Ducker, Langmuir, 2001, 17, 8451-8454.
6 M. Borkovec, I. Szilagyi, I. Popa, M. Finessi, P. Sinha, P. Maroni and G. Papastavrou, Adv. Colloid Interface Sci., 2012, 179-182, 85-98.

7 M. Nayeri, Z. Abbas and J. Bergenholtz, Colloids Surf., A, 2013, 429, 74-81.

8 M. M. Kohonen, M. E. Karaman and R. M. Pashley, Langmuir, 2000, 16, 5749-5753.

9 R. M. Pashley, J. Colloid Interface Sci., 1981, 83, 531-546.

10 G. Toikka, R. A. Hayes and J. Ralston, Langmuir, 1996, 12, 3783-3788.

11 M. Elzbieciak-Wodka, M. Popescu, F. J. Montes Ruiz-Cabello, G. Trefalt, P. Maroni and M. Borkovec, J. Chem. Phys., 2014, 140, 104906.

12 F. J. Montes Ruiz-Cabello, P. Maroni and M. Borkovec, J. Chem. Phys., 2013, 138, 234705.

13 I. Popa, P. Sinha, M. Finessi, P. Maroni, G. Papastavrou and M. Borkovec, Phys. Rev. Lett., 2010, 104, 228301.

14 R. M. Espinosa-Marzal, T. Drobek, T. Balmer and M. P. Heuberger, Phys. Chem. Chem. Phys., 2012, 14, 60856093.

15 M. A. Bevan and D. C. Prieve, Langmuir, 1999, 15, 7925-7936. 16 M. Dishon, O. Zohar and U. Sivan, Langmuir, 2009, 25, 28312836.

17 W. A. Ducker, T. J. Senden and R. M. Pashley, Langmuir, 1992, 8, 1831-1836.

18 K. Hu and A. J. Bard, Langmuir, 1997, 13, 5114-5119.

19 M. Giesbers, J. M. Kleijn and M. A. Cohen Stuart, J. Colloid Interface Sci., 2002, 248, 88-95.

20 J. Morag, M. Dishon and U. Sivan, Langmuir, 2013, 29, 63176322.

21 W. B. Russel, D. A. Saville and W. R. Schowalter, Colloidal Dispersions, Cambridge University Press, Cambridge, 1989.

22 M. Elimelech, J. Gregory, X. Jia and R. A. Williams, Particle Deposition and Aggregation: Measurement, Modeling, and Simulation, Butterworth-Heinemann Ltd., Oxford, 1995.

23 R. Pericet-Camara, G. Papastavrou, S. H. Behrens and M. Borkovec, J. Phys. Chem. B, 2004, 108, 19467-19475.

24 B. V. Zhmud, A. Meurk and L. Bergstrom, J. Colloid Interface Sci., 1998, 207, 332-343.

25 S. Rentsch, H. Siegenthaler and G. Papastavrou, Langmuir, 2007, 23, 9083-9091.

26 S. L. Carnie and D. Y. C. Chan, J. Colloid Interface Sci., 1993, 161, 260-264.

27 S. H. Behrens and M. Borkovec, J. Phys. Chem. B, 1999, 103, 2918-2928.

28 R. M. Pashley, J. Colloid Interface Sci., 1984, 102, 23-35.

29 K. Besteman, M. A. G. Zevenbergen, H. A. Heering and S. G. Lemay, Phys. Rev. Lett., 2004, 93, 170802.

30 O. Zohar, I. Leizerson and U. Sivan, Phys. Rev. Lett., 2006, 96, 177802.

31 M. Dishon, O. Zohar and U. Sivan, Langmuir, 2011, 27, 12977-12984.

32 P. Sinha, I. Szilagyi, F. J. Montes Ruiz-Cabello, P. Maroni and M. Borkovec, J. Phys. Chem. Lett., 2013, 4, 648-652.

33 F. J. Montes Ruiz-Cabello, G. Trefalt, Z. Csendes, P. Sinha, T. Oncsik, I. Szilagyi, P. Maroni and M. Borkovec, J. Phys. Chem. B, 2013, 117, 11853-11862. 
34 D. Ebeling, D. van den Ende and F. Mugele, Nanotechnology, 2011, 22, 305706.

35 R. Kjellander, S. Marcelja, R. M. Pashley and J. P. Quirk, J. Chem. Phys., 1990, 92, 4399-4407.

36 C. Gutsche, U. F. Keyser, K. Kegler and F. Kremer, Phys. Rev. E: Stat., Nonlinear, Soft Matter Phys., 2007, 76, 031403.

37 F. J. Montes Ruiz-Cabello, G. Trefalt, P. Maroni and M. Borkovec, Langmuir, 2014, 30, 4551-4555.

38 J. Forsman, J. Phys. Chem. B, 2004, 108, 9236-9245.

39 M. M. Hatlo and L. Lue, Soft Matter, 2009, 5, 125-133.

40 K. Bohinc, J. M. A. Grime and L. Lue, Soft Matter, 2012, 8, 5679-5686.

41 M. Kanduc, A. Naji, J. Forsman and R. Podgornik, Phys. Rev. E: Stat., Nonlinear, Soft Matter Phys., 2011, 84, 011502.

42 B. I. Shklovskii, Phys. Rev. E: Stat., Nonlinear, Soft Matter Phys., 1999, 60, 5802-5811.

43 G. Tellez, Philos. Trans. R. Soc., A, 2011, 369, 322-334.

44 M. Han and X. J. Xing, J. Stat. Phys., 2013, 151, 1121-1139.

45 R. Kjellander and D. J. Mitchell, J. Chem. Phys., 1994, 101, 603-626.

46 A. McBride, M. Kohonen and P. Attard, J. Chem. Phys., 1998, 109, 2423-2428.

47 G. Trefalt, I. Szilagyi and M. Borkovec, J. Colloid Interface Sci., 2013, 406, 111-120.
48 S. H. Behrens and M. Borkovec, Phys. Rev. E: Stat., Nonlinear, Soft Matter Phys., 1999, 60, 7040-7048.

49 M. Borkovec and S. H. Behrens, J. Phys. Chem. B, 2008, 112, 10795-10799.

50 A. C. Cowley, N. L. Fuller, R. P. Rand and V. A. Parsegian, Biochemistry, 1978, 17, 3163-3168.

51 A. E. Martell, R. M. Smith and R. J. Motekaitis, Critically Selected Stability Constants of Metal Complexes: Version 8.0., National Insitutue of Standards and Technology, Gainesburg, 2004.

52 I. Popa, G. Gillies, G. Papastavrou and M. Borkovec, J. Phys. Chem. B, 2009, 113, 8458-8461.

53 J. E. Sader, J. W. M. Chon and P. Mulvaney, Rev. Sci. Instrum., 1999, 70, 3967-3969.

54 J. L. Hutter and J. Bechhoefer, Rev. Sci. Instrum., 1993, 64, 1868-1873.

55 C. F. Baes and R. E. Mesmer, The Hydrolysis of Cations, Krieger Publishing, Malabar1976.

56 V. Kuznetsov and G. Papastavrou, J. Phys. Chem. C, 2014, 118, 2673-2685.

57 Q. Chen, S. C. Bae and S. Granick, Nature, 2011, 469, 381384.

58 F. Li, D. P. Josephson and A. Stein, Angew. Chem., Int. Ed., 2011, 50, 360-388. 